\title{
PREDICTION OF FRACTIONAL BROWNIAN MOTION WITH HURST INDEX LESS THAN 1/2
}

\author{
V.V. Anh And A. Inoue \\ We give a proof based on an integral equation for an explicit prediction formula for \\ fractional Brownian motion with Hurst index less than 1/2.
}

\section{INTRODUCTION}

A fractional Brownian motion with Hurst index $H \in(0,1)$ is a real centred Gaussian process $\left(B_{H}(t): t \in \mathbf{R}\right)$ with autocovariance

$$
E\left[B_{H}(t) B_{H}(s)\right]=\frac{1}{2}\left(|t|^{2 H}+|s|^{2 H}-|t-s|^{2 H}\right) \quad(t, s \in \mathbf{R}) .
$$

The case $H=1 / 2$ corresponds to the ordinary Brownian motion. Starting from zero, fractional Brownian motion has stationary increments satisfying $E\left[\left(B_{H}(t)-B_{H}(s)\right)^{2}\right]$ $=|t-s|^{2 H}$. For $H \in(0,1) \backslash\{1 / 2\}$, fractional Brownian motion has the following asymptotic behaviour:

$$
\begin{aligned}
E\left[\left\{B_{H}(t+1)-B_{H}(t)\right\}\left\{B_{H}(s+1)-B_{H}(s)\right\}\right] & \\
& \sim H(2 H-1)(t-s)^{2 H-2} \quad(t-s \rightarrow \infty) .
\end{aligned}
$$

Fractional Brownian motion was discovered by Kolmogorov [4] but much of recent works on fractional Brownian motion originate from the seminal paper [6] by Mandelbrot and Van Ness. We refer to Samorodnitsky and Taqqu [9, Sections 7.2 and 14.7] for this background. Fractional Brownian motion has been widely used to model various phenomena in hydrology, network traffic, finance et cetera, which exhibit long-range dependence.

Let $t_{0}, t_{1}$, and $T$ be real constants such that

$$
-\infty<-t_{0} \leqslant 0 \leqslant t_{1}<T<\infty, \quad t_{0}<t_{1} .
$$

The prediction of fractional Brownian motion is concerned with the computation of

$$
E\left[B_{H}(T) \mid \sigma\left(B_{H}(s):-t_{0} \leqslant s \leqslant t_{1}\right)\right]
$$

Received 6th April, 2004

Partially supported by the Australian Research Council grant A10024117.

Copyright Clearance Centre, Inc. Serial-fee code: 0004-9727/04 \$A2.00+0.00. 
and

$$
E\left[B_{H}(T) \mid \sigma\left(B_{H}(s):-\infty<s \leqslant t_{1}\right)\right],
$$

and their representation using $\left(B_{H}(s):-t_{0} \leqslant s \leqslant t_{1}\right)$ and $\left(B_{H}(s):-\infty<s \leqslant t_{1}\right)$, respectively. The first problem is the prediction from a finite part of time, while the second one is the prediction from an infinite part of time. The problem (1.2) with $0<H<1 / 2$ was solved by Yaglom [10], while both problems (1.1) and (1.2) with $1 / 2<H<1$ were solved by Gripenberg and Norros [3]. Nuzman and Poor [8] introduced a new approach based on Lamperti's transformation, and considered all the cases including the remaining problem (1.1) with $0<H<1 / 2$. The present paper gives a new proof based on an integral equation for (1.1) with $0<H<1 / 2$.

The solution of Gripenberg and Norros [3] to (1.1) with $1 / 2<H<1$ is of the form

$$
E\left[B_{H}(T) \mid \sigma\left(B_{H}(s):-t_{0} \leqslant s \leqslant t_{1}\right)\right]=B_{H}\left(t_{1}\right)+\int_{-t_{0}}^{t_{1}} f\left(t_{0}, t_{1}, T, s\right) d B_{H}(s) .
$$

In view of this solution in terms of a stochastic integral with respect to fractional Brownian motion, one tends to believe that the solution to (1.1) with $0<H<1 / 2$ would also be of the form (1.3). However, this is not the case. The solution to (1.1) with $0<H<1 / 2$ is of the form

$$
E\left[B_{H}(T) \mid \sigma\left(B_{H}(s):-t_{0} \leqslant s \leqslant t_{1}\right)\right]=\int_{-t_{0}}^{t_{1}} f\left(t_{0}, t_{1}, T, s\right) B_{H}(s) d s,
$$

that is, in terms of an elementary integral.

In our method for obtaining a solution to (1.1) with $0<H<1 / 2$ of the form (1.4), we reduce the problem to a manageable computation by the following equality for $f$ in (1.4):

$$
\int_{-t_{0}}^{t_{1}} f\left(t_{0}, t_{1}, T, s\right) d s=1
$$

It is found in [1] that the same equality as (1.5) holds for more general processes than fractional Brownian motion with $0<H<1 / 2$.

The solution to (1.1) in the case $0<H<1 / 2$ is given by the following theorem.

THEOREM 1. Let $t_{0}, t_{1}$ and $T$ be as above. We assume $0<H<1 / 2$. Then

$$
\begin{aligned}
& E\left[B_{H}(T) \mid \sigma\left(B_{H}(s):-t_{0} \leqslant s \leqslant t_{1}\right)\right] \\
& =\frac{\sin (\pi((1 / 2)-H))}{\pi} \int_{-t_{0}}^{t_{1}}\left(\frac{T-t_{1}}{t_{1}-s}\right)^{(1 / 2)+H}\left(\frac{t_{0}+s}{T+t_{0}}\right)^{(1 / 2)-H} \frac{1}{T-s} B_{H}(s) d s \\
& +\frac{((1 / 2)-H) \sin (\pi((1 / 2)-H))}{\pi\left(t_{0}+t_{1}\right)}\left[\int_{0}^{\left(T-t_{1}\right) /\left(T+t_{0}\right)} u^{H-(1 / 2)}(1-u)^{-2 H} d u\right] \\
& \times \int_{-t_{0}}^{t_{1}}\left[\left(\frac{t_{0}+t_{1}}{t_{0}+s}\right)\left(\frac{t_{0}+t_{1}}{t_{1}-s}\right)\right]^{H+(1 / 2)} B_{H}(s) d s .
\end{aligned}
$$


The solution to (1.1) with $0<H<1 / 2$ was first obtained by Nuzman and Poor [8], and the theorem above is essentially the same as their result (Theorem 4.4 in [8]) except for one point. Unlike the theorem above, they do not assume that the interval corresponding to $\left[-t_{0}, t_{1}\right]$ includes the origin. However, their argument on this point does not seem to be complete. In our notation, they claimed that observation of $B_{H}(t)$ on $\left[-t_{0}, t_{1}\right]$ is equivalent to that of $B_{H}\left(t-t_{0}\right)-B_{H}\left(-t_{0}\right)$ on $\left[0, t_{0}+t_{1}\right]$ (see Section 3.3 in [8]). However, this is true only if $B_{H}\left(-t_{0}\right)$ is a priori known, that is, $t_{0}=0$, and thus $B_{H}\left(-t_{0}\right)=0$. We also remark that, in $\left[8\right.$, Theorem 4.4], the factor $\pi^{-1} \sin (\pi((1 / 2)-H))$ or $\pi^{-1} \cos (\pi H)$ is missing.

In [3], the solution to (1.1) with $1 / 2<H<1$ is obtained by reducing the problem to the following type of singular integral equation for the prediction kernel:

$$
\int_{0}^{1} F(t)|s-t|^{-\alpha} d t=f(s) \quad(0<s<1), \quad 0<\alpha<1 .
$$

In this paper, we obtain (1.6) by reducing the problem to the following type of equation (that is, (2.9) below) for the prediction kernel:

$$
\int_{0}^{1} F(t)|s-t|^{-\alpha} \operatorname{sgn}(s-t) d t=f(s) \quad(0<s<1), \quad 0<\alpha<1 .
$$

It is interesting to note that both (1.7) and (1.8) were solved in the same paper by Lundgren and Chiang [5] (though the solution to the first equation (1.7) had already been given by Carleman [2]).

\section{PROOF OF THEOREM 1}

As stated in Section 1, we look for a nonnegative measurable function $h(t)$ $=h\left(t ; t_{2}, t_{3}\right)$ on $\left(0, t_{2}\right)$ satisfying

$$
\begin{aligned}
\int_{0}^{t_{2}} h(t) d t & =1 \\
E\left[B_{H}(T) \mid \sigma\left(B_{H}(s):-t_{0} \leqslant s \leqslant t_{1}\right)\right] & =\int_{-t_{0}}^{t_{1}} h\left(t+t_{0}\right) B_{H}(t) d t
\end{aligned}
$$

where the positive constants $t_{2}$ and $t_{3}$ are defined by

$$
t_{2}:=t_{0}+t_{1}, \quad t_{3}:=T-t_{1} .
$$

These facts are essential in deriving the manageable form (1.8), hence finally obtaining (1.6).

The equality (2.2) implies

$$
E\left[\left\{B(T)-\int_{-t_{0}}^{t_{1}} h\left(t+t_{0}\right) B_{H}(t) d t\right\} B_{H}(s)\right]=0 \quad\left(-t_{0}<s<t_{1}\right)
$$


or

$$
\begin{aligned}
\int_{-t_{0}}^{t_{1}} h\left(t+t_{0}\right)\left(|t|^{2 H}+|s|^{2 H}-\mid s-\right. & \left.\left.t\right|^{2 H}\right) d t \\
& =T^{2 H}+|s|^{2 H}-(T-s)^{2 H} \quad\left(-t_{0}<s<t_{1}\right) .
\end{aligned}
$$

From this and (2.1), we obtain

$$
\int_{-t_{0}}^{t_{1}} h\left(t+t_{0}\right)\left(|t|^{2 H}-|s-t|^{2 H}\right) d t=T^{2 H}-(T-s)^{2 H} \quad\left(-t_{0}<s<t_{1}\right) .
$$

Since we have, for $-t_{0}<s<t_{1}$,

$$
\int_{-t_{0}}^{t_{1}} h\left(t+t_{0}\right)|s-t|^{2 H} d t=\int_{-t_{0}}^{s} h\left(t+t_{0}\right)(s-t)^{2 H} d t+\int_{s}^{t_{1}} h\left(t+t_{0}\right)(t-s)^{2 H} d t
$$

formal differentiation of both sides of (2.5) with respect to $s$ yields

$$
\int_{-t_{0}}^{t_{1}} h\left(t+t_{0}\right)|s-t|^{2 H-1} \operatorname{sgn}(s-t) d t=-(T-s)^{2 H-1} \quad\left(-t_{0}<s<t_{1}\right)
$$

(the validity of this formal calculation should not be of concern at this stage). We define $\alpha \in(0,1), a \in(0, \infty)$, and $g(t)=g\left(t ; t_{2}, t_{3}\right)$, respectively, by

$$
\begin{aligned}
\alpha & :=1-2 H, \quad a:=t_{3} / t_{2}, \\
g(t) & :=t_{2} h\left(t_{2}(1-t)\right) \quad(0<t<1) .
\end{aligned}
$$

Then, by the substitutions $t^{\prime}=\left(t_{1}-t\right) / t_{2}$ and $s^{\prime}=\left(t_{1}-s\right) / t_{2}$, we obtain

$$
\int_{0}^{1} g(t)|s-t|^{-\alpha} \operatorname{sgn}(s-t) d t=(a+s)^{-\alpha} \quad(0<s<1) .
$$

By $[5,(18)]$, the general solution $g(t)$ to $(2.9)$ is given by

$$
g(t)=c_{1} t^{(\alpha / 2)-1}(1-t)^{(\alpha / 2)-1}+g_{0}(t) \quad(0<t<1),
$$

where $c_{1}$ is an arbitrary constant and $g_{0}(t)$ is given by, for $0<t<1$,

$$
\frac{\Gamma(\alpha) \sin (\pi \alpha / 2)}{\pi \Gamma(\alpha / 2)^{2}} \frac{d}{d t} t^{\alpha / 2} \int_{t}^{1} s^{-\alpha}(s-t)^{(\alpha / 2)-1}\left\{\int_{0}^{s} u^{\alpha / 2}(s-u)^{(\alpha / 2)-1}(a+u)^{-\alpha} d u\right\} d s
$$

By the change of variables $v=u / s$, we have

$$
\begin{aligned}
\int_{0}^{s} u^{\alpha / 2}(s-u)^{(\alpha / 2)-1}(a+u)^{-\alpha} d u=\left(\frac{s}{a}\right)^{\alpha} & \int_{0}^{1} v^{\alpha / 2}(1-v)^{(\alpha / 2)-1}\left(1+\frac{s}{a} v\right)^{-\alpha} d v \\
& =\frac{\Gamma(\alpha / 2)^{2}}{2 \Gamma(\alpha)}\left(\frac{s}{a}\right)^{\alpha} F\left(\alpha, \frac{\alpha}{2}+1 ; \alpha+1 ;-\frac{s}{a}\right)
\end{aligned}
$$


where $F(a, b ; c ; z)={ }_{2} F_{1}(a, b ; c ; z)$ is the hypergeometric function. Thus, for $0<t<1$,

$$
g_{0}(t)=\frac{\sin (\pi \alpha / 2)}{2 \pi} \frac{d}{d t} t^{\alpha / 2} \int_{t}^{1} s^{-\alpha}(s-t)^{(\alpha / 2)-1}\left(\frac{s}{a}\right)^{\alpha} F\left(\alpha, \frac{\alpha}{2}+1 ; \alpha+1 ;-\frac{s}{a}\right) d s
$$

From (2.11), we easily find that $\int_{0}^{1} g_{0}(t) d t=0$. Since we have $\int_{0}^{t_{2}} h(t) d t$ $=\int_{0}^{1} g(t) d t$, the condition (2.1) implies

$$
c_{1}=\frac{\Gamma(\alpha)}{\Gamma(\alpha / 2)^{2}}
$$

We now obtain an explicit expression for $g_{0}(t)$ using (2.11). By the formulas

$$
\begin{aligned}
\frac{d}{d z}\left[z^{a} F(a, b ; c ; z)\right] & =a z^{a-1} F(a+1, b ; c ; z), \\
F(a, b ; c ; z) & =(1-z)^{c-a-b} F(c-a, c-b ; c ; z),
\end{aligned}
$$

we see that

$$
\frac{d}{d s}\left[\left(\frac{s}{a}\right)^{\alpha} F\left(\alpha, \frac{\alpha}{2}+1 ; \alpha+1 ;-\frac{s}{a}\right)\right]=\frac{\alpha}{a}\left(\frac{s}{a}\right)^{\alpha-1}\left(1+\frac{s}{a}\right)^{-(\alpha / 2)-1} .
$$

On the other hand, by the change of variables $v=(u-t) / t$, we have

$$
t^{\alpha / 2} \int_{t}^{s} u^{-\alpha}(u-t)^{(\alpha / 2)-1} d u=f\left(\frac{s-t}{t}\right), \quad(t \leqslant s \leqslant 1)
$$

where

$$
f(x):=\int_{0}^{x}(1+v)^{-\alpha} v^{(\alpha / 2)-1} d v \quad(x \geqslant 0) .
$$

We have

$$
\frac{d}{d t} f\left(\frac{s-t}{t}\right)=-t^{(\alpha / 2)-1} s^{1-\alpha}(s-t)^{(\alpha / 2)-1} \quad(0<t<s) .
$$

Hence, by integration by parts, we obtain, for $0<t<1$,

$$
g_{0}(t)=-\frac{\sin (\pi \alpha / 2)}{2 \pi} a^{-\alpha} F\left(\alpha, \frac{\alpha}{2}+1 ; \alpha+1 ;-\frac{1}{a}\right) t^{(\alpha / 2)-1}(1-t)^{(\alpha / 2)-1}+g_{1}(t),
$$

where

$$
\begin{aligned}
g_{1}(t) & =-\frac{\sin (\pi \alpha / 2)}{2 \pi} \frac{d}{d t} \int_{t}^{1} f\left(\frac{s-t}{t}\right) \frac{\alpha}{a}\left(\frac{s}{a}\right)^{\alpha-1}\left(1+\frac{s}{a}\right)^{-(\alpha / 2)-1} d s \\
& =-\frac{\sin (\pi \alpha / 2)}{2 \pi} \int_{t}^{1}\left\{\frac{d}{d t} f\left(\frac{s-t}{t}\right)\right\} \frac{\alpha}{a}\left(\frac{s}{a}\right)^{\alpha-1}\left(1+\frac{s}{a}\right)^{-(\alpha / 2)-1} d s \\
& =\frac{\alpha \sin (\pi \alpha / 2)}{2 \pi}\left(\frac{a}{t}\right)^{1-(\alpha / 2)} \int_{t}^{1}(s-t)^{(\alpha / 2)-1}(a+s)^{-(\alpha / 2)-1} d s
\end{aligned}
$$

By the change of variables $u=(1-s) /(1-t)$ and the equality

$$
\int_{0}^{1} t^{\mu-1}(1-t)^{\nu-1}(c-t)^{-\mu-\nu} d t=c^{-\nu}(c-1)^{-\mu} B(\mu, \nu) \quad(\mu, \nu>0, c>1)
$$


(see $[7$, Lemma $2.2(i)]$ ), we see that, for $0<t<1$,

$$
\begin{aligned}
g_{1}(t) & =\frac{\alpha \sin (\pi \alpha / 2)}{2 \pi}\left(\frac{a}{t}\right)^{1-(\alpha / 2)} \frac{1}{1-t} \int_{0}^{1}(1-u)^{(\alpha / 2)-1}\left(\frac{a+1}{1-t}-u\right)^{-(\alpha / 2)-1} d u \\
& =\frac{\sin (\pi \alpha / 2)}{\pi}\left(\frac{a}{t}\right)^{1-(\alpha / 2)}\left(\frac{1-t}{a+1}\right)^{\alpha / 2} \frac{1}{a+t} .
\end{aligned}
$$

From this as well as (2.10) and (2.13), the general solution $g(t)$ to the equation (2.9) is given by

$$
g(t)=c_{2} t^{(\alpha / 2)-1}(1-t)^{(\alpha / 2)-1}+\frac{\sin (\pi \alpha / 2)}{\pi}\left(\frac{a}{t}\right)^{1-(\alpha / 2)}\left(\frac{1-t}{a+1}\right)^{\alpha / 2} \frac{1}{a+t},
$$

for $0<t<1$, where $c_{2}$ is an arbitrary constant.

By (2.12), under the condition (2.1), $c_{2}$ is given by

$$
c_{2}:=\frac{\Gamma(\alpha)}{\Gamma(\alpha / 2)^{2}}-\frac{\sin (\pi \alpha / 2)}{2 \pi} a^{-\alpha} F\left(\alpha, \frac{\alpha}{2}+1 ; \alpha+1 ;-\frac{1}{a}\right) .
$$

However, since we have, for $0<\mu<1, \nu>0$ and $c>1$,

$$
\begin{aligned}
\int_{0}^{1} t^{\mu-1}(1-t)^{\nu-1}(c-t)^{-\mu-\nu+1} d t & \\
= & \frac{\pi}{\sin (\pi \mu)}-(\mu+\nu-1) B(\mu, \nu) \int_{0}^{1-(1 / c)} s^{-\mu}(1-s)^{\mu+\nu-2} d s
\end{aligned}
$$

(see [7, Lemma 2.2 (iii)]), we see that

$$
\begin{aligned}
c_{2} & =\frac{\Gamma(\alpha)}{\Gamma(\alpha / 2)^{2}}\left[1-\frac{\sin (\pi \alpha / 2)}{\pi} \int_{0}^{1} s^{(\alpha / 2)-1}(1-s)^{\alpha / 2}(a+1-s)^{-\alpha} d s\right] \\
& =\frac{\alpha \sin (\pi \alpha / 2)}{2 \pi} \int_{0}^{a /(1+a)} s^{-\alpha / 2}(1-s)^{\alpha-1} d s .
\end{aligned}
$$

Thus, using (2.7) and (2.8), we finally obtain, for $-t_{0}<t<t_{1}$,

$$
\begin{array}{r}
h(t)=\frac{\sin (\pi((1 / 2)-H))}{\pi}\left(\frac{T-t_{1}}{t_{2}-t}\right)^{(1 / 2)+H}\left(\frac{t}{T+t_{0}}\right)^{(1 / 2)-H} \frac{1}{T+t_{0}-t} \\
+\frac{((1 / 2)-H) \sin (\pi((1 / 2)-H))}{\pi t_{2}}\left[\int_{0}^{\left(T-t_{1}\right) /\left(T+t_{0}\right)} u^{H-(1 / 2)}(1-u)^{-2 H} d u\right] \\
\times\left[\left(\frac{t_{2}}{t}\right)\left(\frac{t_{2}}{t_{2}-t}\right)\right]^{H+(1 / 2)}
\end{array}
$$

which implies (1.6).

Now, for a rigorous proof, we may start with (2.14). Then we have (2.6) and (2.1) by the arguments given above. From (2.6), we see (rigorously this time) that

$$
\frac{d}{d s} \phi(s)=2(T-s)^{2 H-1} H \quad\left(-t_{0}<s<t_{1}\right)
$$


where

$$
\phi(s):=\int_{-t_{0}}^{t_{1}} h\left(t+t_{0}\right)\left(|t|^{2 H}-|s-t|^{2 H}\right) d t \quad(s \in \mathbf{R}) .
$$

Since $\phi(0)=0$, we get (2.5). Finally, from (2.5) and (2.1), we obtain (2.4) or (2.3) or (1.6). This completes the proof of Theorem 1.

\section{REMARKS}

1. From the proof in Section 2, we see that

$$
\int_{0}^{1} g_{1}(t)|s-t|^{-\alpha} \operatorname{sgn}(s-t) d t=(a+s)^{-\alpha} \quad(0<s<1) .
$$

This implies the following equality: for $a>0,0<\alpha<1$ and $0<s<1$,

$$
\int_{0}^{1} \frac{t^{(\alpha / 2)-1}(1-t)^{\alpha / 2}}{a+t}|s-t|^{-\alpha} \operatorname{sgn}(s-t) d t=\frac{\pi}{\sin (\pi \alpha / 2)} a^{(\alpha / 2)-1}(a+1)^{\alpha / 2}(a+s)^{-\alpha}
$$

2. Since $E\left[B_{H}(s)^{2}\right]^{1 / 2}=|s|^{H}$, we easily find that the second term on the right-hand side of (1.6) tends to zero, as $t_{0} \rightarrow \infty$, in $L^{2}(\Omega, \mathcal{F}, P)$, where $(\Omega, \mathcal{F}, P)$ is the probability space on which $\left(B_{H}(t)\right)$ is defined. Hence, by letting $t_{0} \rightarrow \infty$ in (1.6), we obtain the following prediction formula for fractional Brownian motion with $H \in(0,1 / 2)$ from the infinite past:

$$
\begin{aligned}
E\left[B_{H}(T) \mid \sigma\left(B_{H}(s):\right.\right. & \left.\left.-\infty<s \leqslant t_{1}\right)\right] \\
& =\frac{\sin (\pi((1 / 2)-H))}{\pi} \int_{-\infty}^{t_{1}}\left(\frac{T-t_{1}}{t_{1}-s}\right)^{(1 / 2)+H} \frac{1}{T-s} B_{H}(s) d s
\end{aligned}
$$

This result was given in Yaglom [10, (3.41)].

\section{REFERENCES}

[1] V.V. Anh and A. Inoue, 'Prediction of fractional Brownian motion-type processes: the case $0<H<1 / 2$ ', (submitted).

[2] T. Carleman, 'Über die Abelsche Intergralgleichung mit konstanten Integrations-grenzen', Math. Z. 15 (1922), 111-120.

[3] G. Gripenberg and I. Norros, 'On the prediction of fractional Brownian motion', J. Appl. Probab. 33 (1996), 400-410.

[4] A.N. Kolmogorov, 'Wienersche Spiralen und einige andere interessante Kurven im Hilbertschen raum', Compres Rendus (Doklady) de l'Académie des Sciences de l'USSR (N.S.) 26 (1940), 115-118.

[5] T. Lundgren and D. Chiang, 'Solution of a class of singular integral equations', Quart. Appl. Math. 24 (1967), 303-313.

[6] B.B. Mandelbrot and J.W. Van Ness, 'Fractional Brownian motions, fractional noises and applications', SIAM Rev. 10 (1968), 422-437. 
[7] I. Norros, E. Valkeila, and J. Virtamo, 'An elementary approach to a Girsanov formula and other analytical results on fractional Brownian motions', Bernoulli 5 (1999), 571-587.

[8] C.J. Nuzman and H.V. Poor, 'Linear estimation of self-similar processes via Lamperti's transformation', J. Appl. Probab. 37 (2000)), 429-452.

[9] G. Samorodnitsky and M.S. Taqqu, Stable non-Gaussian random processes: stochastic models with infinite variance (Chapman and Hall, New York, 1994).

[10] A.M. Yaglom, 'Correlation theory of processes with random stationary $n$th increments', (Russian), Mat. Sb. N.S. 37 (1955), 141-196. English translation: Amer. Math. Soc. Trans. Ser. (2) 8 (1958), 87-141.

School of Mathematical Sciences

Queensland University of Technology

GPO Box 2434, Brisbane, Queensland 4001

Australia

e-mail: v.anh@qut.edu.au
Department of Mathematics

Faculty of Science

Hokkaido University

Sapporo 060-0810

Japan

e-mail: inoue@math.sci.hokudai.ac.jp 